Acta Zoológica Mexicana (nueva serie), Volumen 37, 1-23.

https://doi.org/10.21829/azm.2021.3712362

Artículo original

\title{
Las arañas (Arachnida: Araneae) del estado de Hidalgo, México: contribución al conocimiento de su biodiversidad
}

\author{
The spiders (Arachnida: Araneae) from \\ Hidalgo state, Mexico: contribution to \\ knowledge of its biodiversity
}

\section{Miguel OROZCO-GIL, (iD 2*Marco ANTONIO DESALES-LARA}

${ }^{1}$ Centro Universitario de Ciencias Biológicas y Agropecuarias, Universidad de Guadalajara. Camino Ramón Padilla Sánchez No. 2100. C.P. 45200, Zapopan, Jalisco, México.

${ }^{2}$ Facultad de Ciencias Agrícolas, Universidad Autónoma del Estado de México (UAEMéx), Campus El Cerrillo. Carretera Toluca-Ixtlahuaca Kilómetro 15.5, El Cerrillo, Piedras Blancas, C.P. 50200, Toluca, Estado de México, México.

Editor responsable: María Luisa Jiménez-Jiménez

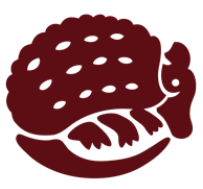

OPEN ACCESS

*Autor corresponsal:

Marco Antonio Desales-Lara madesales@gmail.com

Cita:

Orozco-Gil, M., Desales-Lara, M. A.

(2021) Las arañas (Arachnida: Araneae) del estado de Hidalgo,

México: contribución al conocimiento de su biodiversidad.

Acta Zoológica Mexicana (nueva serie), 37, 1-23.

10.21829/azm.2021.3712362 elocation-id: e3712362

Recibido: 15 octubre 2020 Aceptado: 23 marzo 2021

Publicado: 24 mayo 2021
RESUMEN. En México existen pocas o nulas colecciones en instituciones dedicadas al estudio de las arañas. Esto se refleja en el limitado conocimiento sobre su biodiversidad, pues solo nueve entidades federativas de 32 tienen documentada su araneofauna. En Hidalgo, el número de especies de arañas solo se conoce a partir de dos recopilaciones bibliográficas sobre la biodiversidad de arañas en México. Por lo anterior, el presente trabajo se orientó en ampliar el conocimiento de las arañas de esta entidad a través de una exhaustiva revisión bibliográfica y la examinación del material depositado en la Colección de Arácnidos del Estado de México (CAEM) de la Facultad de Ciencias de la Universidad Autónoma del Estado de México (UAEMéx). Se encontró un nuevo registro para México (Anyphaena pacifica) y diez nuevos registros para el estado de Hidalgo (Anyphaena cf. hespar, Eustala rosae, Habronattus oregonensis, Ixchela abernathyi, Mecaphesa decora, Oxyopes salticus, Paraphidippus aurantius, Phidippus carneus, Steatoda grossa y Theridion niveum). Se proporciona un listado faunístico que consta de 207 especies de arañas, agrupadas en 101 géneros y 33 familias. Las familias con mayor número de géneros y especies fueron: Theridiidae, Araneidae, Lycosidae, 
Salticidae y Gnaphosidae. Con el presente trabajo, Hidalgo pasó del décimo quinto lugar al octavo sitio en cuanto a diversidad de arañas en México.

Palabras clave: araneofauna; diversidad; lista de especies; nuevos registros; colección

ABSTRACT. In Mexico there are few collections and institutions dedicated to the study of spiders. This has limited the knowledge about its biodiversity, because only nine states of 32 have documented its spider diversity. In Hidalgo state, the number of spider species is only known from two bibliographic compilations about the biodiversity of spiders in Mexico. Therefore, the present work aims to expand the knowledge of the spiders of this entity through an exhaustive bibliographic review and the revision of specimens deposited in the Arachnid Collection of the State of Mexico (CAEM) of the Sciences Faculty of the Autonomous University of the State of Mexico (UAEMex). One new record was found for Mexico (Anyphaena pacifica) and ten new records for Hidalgo State (Anyphaena cf. hespar, Eustala rosae, Habronattus oregonensis, Ixchela abernathyi, Mecaphesa decora, Oxyopes salticus, Paraphidippus aurantius, Phidippus carneus, Steatoda grossa and Theridion niveum). A checklist is provided consisting of 207 spider species, grouped into 101 genera and 33 families. The families with the highest number of genera and species were: Theridiidae, Araneidae, Lycosidae, Salticidae and Gnaphosidae. With the present work, Hidalgo went from the fifteenth to eighth place in terms of spider diversity in Mexico.

Key words: araneofauna; diversity; species list; new records; collection

\section{INTRODUCCIÓN}

Dentro de los artrópodos, el orden Araneae es el séptimo grupo más diverso (Coddington \& Levi, 1991) con 49,084 especies descritas, las cuales se agrupan en 4,205 géneros y 128 familias (World Spider Catalog, 2021). Las arañas son conocidas por su capacidad de producir seda para elaborar telarañas, madrigueras, trampas y ovisacos, además de que el veneno de algunas especies es tóxico para el ser humano (Craig, 2003; Foelix, 2011). Estos organismos son de hábitos depredadores y su dieta se basa principalmente en insectos, por lo tanto, son aliadas naturales del ser humano en el manejo de aquellos insectos considerados nocivos (Polis \& Hurd, 1995; IbarraNúñez, 2011). Además, las arañas se encuentran ampliamente distribuidas por todo el mundo en cualquier ecosistema terrestre (Hoffmann, 1993; Platnick, 2020) y ocupan un lugar importante dentro de las redes tróficas, ya que presentan una compleja red de interacciones con el entorno y otras especies (Jiménez, 1996; Ubick et al., 2017). Su amplia distribución en los ecosistemas y su elevado número de especies hacen justificable el estudio de este grupo en diferentes perspectivas (Coddington \& Levi, 1991; Foelix, 2011).

En México existen al menos ocho colecciones o instituciones en las que se realizan investigaciones sobre taxonomía, sistemática y ecología de arañas (Desales-Lara et al., 2018) y sus resultados han contribuido a que en el país se registren 2,388 especies (Durán-Barrón et al., 2016). Este número se ha incrementado con los trabajos de Maya-Morales y Jiménez (2017); ValdezMondragón (2017); Campuzano y Ibarra-Núñez (2018); Chamé-Vázquez et al. (2018), Desales-Lara et al. (2018), Mendoza y Francke (2018), Salgueiro-Sepúlveda y Álvarez-Padilla (2018), Valdez- 
Mondragón et al. (2018), Chamé-Vázquez y Ibarra-Núñez (2019), Guerrero-Fuentes y Francke (2019), Rivera-Quiroz y Álvarez-Padilla (2019), Valdez-Mondragón et al. (2019), Guerrero-Fuentes y Desales-Lara (2020), Jiménez et al. (2020), Navarro-Rodríguez y Valdez-Mondragón (2020) y Valdez-Mondragón (2020). Pese a los esfuerzos realizados, el conocimiento sobre la biodiversidad de la araneofauna de México aún es limitado, las únicas entidades federativas que tienen documentada su diversidad de arañas son: Chiapas (487 especies) (García-Villafuerte, 2009; IbarraNúñez, 2013), Ciudad de México (127 especies) (Durán-Barrón et al., 2016), Coahuila (178 especies) (Desales-Lara et al., 2018), Estado de México (215 especies) (Desales-Lara, 2014; Guerrero-Fuentes \& Desales-Lara, 2020), Michoacán (89 especies) (Ponce, 2005), Quintana Roo (184 especies) (SalasSuárez \& Beutelspacher, 2011), San Luis Potosí (435 especies) (Montaño-Moreno \& Lucio-Palacio, 2019), Tamaulipas (350 especies) (Gómez-Rodríguez et al., 2014) y Veracruz (373 especies) (IbarraNúñez, 2011).

En Hidalgo no existe una colección de arácnidos, y esto se ve reflejado en el actual desconocimiento de la biodiversidad de este grupo. El número de especies de arañas presentes en esta entidad solo se conoce a partir de dos recopilaciones bibliográficas que engloban la diversidad de arañas en México. La primera fue la de Hoffmann (1976) quien enlistó 76 especies de arañas para el estado y, posteriormente, Jiménez (1996) indicó únicamente 125 especies para la entidad, sin incluir más datos. Por lo tanto, el objetivo del presente trabajo es contribuir al conocimiento de la biodiversidad de arañas del estado de Hidalgo, presentar el primer listado de especies actualizado para la entidad y dar a conocer nuevos registros de especies de arañas para el estado y el país.

\section{MATERIALES Y MÉTODOS}

Se determinaron las arañas del estado de Hidalgo depositadas en la Colección de Arácnidos del Estado de México (CAEM) de la Facultad de Ciencias de la Universidad Autónoma del Estado de México (UAEMéx). De acuerdo con los datos de recolecta, las arañas se capturaron entre septiembre y octubre de 2007 en tres localidades distintas del municipio de Tepeji del Río de Ocampo, Hidalgo, México. Este municipio se ubica dentro de la Provincia Biogeográfica del Eje Volcánico Transmexicano (EVT) en la Zona de Transición Mexicana (ZTM). Cuenta con un clima templado subhúmedo con lluvias en verano y los tipos de vegetación que más predominan son los pastizales y matorrales (Morrone, 2005; INEGI, 2009). Las arañas adultas fueron determinadas a nivel de especie con ayuda de un microscopio estereoscópico Labomed ${ }^{\circledR}$ modelo CZM4, y claves especializadas de diversos autores, mientras que los especímenes juveniles solo fueron determinados a nivel de familia con la clave de Ubick et al. (2017). Algunos genitales de los ejemplares adultos fueron disectados $\mathrm{y}$, posteriormente, reblandecidos con $\mathrm{KOH}$ al $10 \%$ y aclarados con ácido láctico para la determinación a nivel especifico (Ubick et al., 2017). Las identificaciones de todos los especímenes fueron corroboradas por ambos autores. Los nuevos registros fueron ilustrados con fotografías digitales tomadas con la técnica multifocal con una cámara Olympus ${ }^{\circledR}$ modelo DSC-F828 de 8.0 megapíxeles, la cual se encontraba acoplada a un microscopio estereoscópico Olympus ${ }^{\circledR}$ modelo SZ2-ILST. Las fotografías se manipularon con Adobe Photoshop CS6. En un mismo frasco se encontraron depositados más de un ejemplar de la misma especie, por lo tanto, un mismo código fue asignado para varios organismos. 
La actualización del listado de especies se llevó a cabo teniendo como base la recopilación bibliográfica de Hoffmann (1976). Posteriormente, se complementó con una extensa revisión bibliográfica de artículos científicos donde se citaban especies de arañas para México, de los cuales se tomaron los registros para el estado de Hidalgo. Las especies encontradas se validaron con el World Spider Catalog (2021) y los cambios nomenclaturales y sinonimias fueron considerados en el listado de especies.

\section{RESULTADOS \\ Arañas de Hidalgo}

En la CAEM se encontraron depositados 245 ejemplares $(42 \hat{\jmath} \hat{\jmath}, 98$ 우 y 105 juveniles) del estado de Hidalgo, los cuales se agruparon en 15 familias, 23 géneros y 30 especies. La familia Araneidae tuvo el mayor número de ejemplares (106) y especies (cinco), seguida de Salticidae (34 ejemplares y tres especies). En contraste, la familia Nesticidae solo estuvo representada por dos ejemplares y una especie (Fig. 1). De las 30 especies encontradas en la CAEM, una es un nuevo registro para México y diez son nuevos registros para el estado de Hidalgo.

De los 973 trabajos consultados, solo en 55 se encontraron registros de especies de arañas para Hidalgo, de los cuales el $87 \%$ fueron realizados por aracnólogos extranjeros y el 23\% restante por de aracnólogos mexicanos. Con base en la revisión bibliográfica y los nuevos registros del presente trabajo, se enlistan 207 especies de arañas para el estado de Hidalgo, las cuales se agrupan en 101 géneros y 33 familias (Cuadro 1). Las familias con mayor número de géneros y especies para Hidalgo fueron: Theridiidae (19 géneros y 47 especies), seguida de Araneidae (13 géneros y 27 especies), Lycosidae (siete géneros y 19 especies), Salticidae (seis géneros y 16 especies) y Gnaphosidae (cinco géneros y 13 especies) (Fig. 2). El resto de las familias tienen cinco o menos géneros y de una a diez especies registradas (Cuadro 1).

Cuadro 1. Familias, géneros y especies presentes en el estado de Hidalgo.

\begin{tabular}{ll}
\hline Familia & Género y/o especie \\
\hline Agelenidae & Agelenopsis aperta Gertsch, 1934 \\
& Eratigena guanato Bolzern \& Hänggi, 2016 \\
& Eratigena mexicana Roth, 1968 \\
& Eratigena rothi Gertsch, 1971 \\
& Eratigena selva Roth, 1968 \\
& Eratigena tlaxcala Roth, 1968 \\
& Eratigena xilitla Bolzern \& Hänggi, 2016 \\
& Hoffmannilena tizayuca Maya-Morales \& Jiménez, 2016 \\
& Tegenaria domestica Clerck, 1757 \\
& Anyphaena catalina Platnick, 1974 \\
& Anyphaena cielo Platnick \& Lau, 1975 \\
\hline Anyphaenidae & Anyphaena cf. hespar Platnick, 1974 \\
& Anyphaena judicata O. Pickard-Cambridge, 1896 \\
& Anyphaena pacifica Banks, 1896 \\
& Anyphaena tehuacan Platnick \& Lau, 1975
\end{tabular}




\begin{tabular}{|c|c|}
\hline Familia & Género y/o especie \\
\hline & Hibana incursa Chamberlin, 1919 \\
\hline \multirow[t]{27}{*}{ Araneidae } & Allocyclosa bifurca McCook, 1887 \\
\hline & Argiope argentata Fabricius, 1775 \\
\hline & Argiope blanda O. Pickard-Cambridge, 1898 \\
\hline & Argiope trifasciata Forsskål, 1775 \\
\hline & Araneus guerrerensis Chamberlin \& Ivie, 1936 \\
\hline & Araneus sinistrellus Roewer, 1942 \\
\hline & Cyclosa berlandi Levi, 1999 \\
\hline & Eriophora edax Blackwall, 1863 \\
\hline & Eustala californiensis Keyserling, 1885 \\
\hline & Eustala rosae Chamberlin \& Ivie, 1935 \\
\hline & Eustala scitula Chickering, 1955 \\
\hline & Kaira altiventer O. Pickard-Cambridge, 1889 \\
\hline & Larinia directa Hentz, 1847 \\
\hline & Mangora bimaculata O. Pickard-Cambridge, 1889 \\
\hline & Magnora calcalifera F. O. Pickard-Cambridge, 1904 \\
\hline & Mangora fascialata Franganillo, 1936 \\
\hline & Mangora passiva O. Pickard-Cambridge, 1889 \\
\hline & Magnora picta O. Pickard-Cambridge, 1889 \\
\hline & Metazygia zilloides Banks, 1898 \\
\hline & Metepeira atascadero Piel, 2001 \\
\hline & Metepeira incrassata F. O. Pickard-Cambridge, 1903 \\
\hline & Metepeira spinipes F. O. Pickard-Cambridge, 1903 \\
\hline & Neoscona arabesca Walckenaer, 1841 \\
\hline & Neoscona crucifera Lucas, 1838 \\
\hline & Neoscona oaxacensis Keyserling, 1864 \\
\hline & Neoscona orizabensis F. O. Pickard-Cambridge, 1904 \\
\hline & Ocrepeira rufa O. Pickard-Cambridge, 1889 \\
\hline \multirow[t]{3}{*}{ Cheiracanthiidae } & Strotarchus jacala Bonaldo, Saturnino, Ramírez \& Brescovit, 2012 \\
\hline & Strotarchus piscatorius Hentz, 1847 \\
\hline & Strotarchus tlaloc Bonaldo, Saturnino, Ramírez \& Brescovit, 2012 \\
\hline Clubionidae & Elaver cf. tigrina O. Pickard-Cambridge, 1898 \\
\hline Corinnidae & Mazax pax Reiskind, 1969 \\
\hline \multirow[t]{6}{*}{ Dictynidae } & Dictyna jacalana Gertsch \& Davis, 1937 \\
\hline & Mallos niveus O. Pickard-Cambridge, 1902 \\
\hline & Mexitlia altima Bond \& Opell, 1997 \\
\hline & Mexitlia grandis O. Pickard-Cambridge, 1896 \\
\hline & Mexitlia trivittata Banks, 1901 \\
\hline & Phantyna mulegensis Chamberlin, 1924 \\
\hline \multirow[t]{2}{*}{ Euagridae } & Euagrus charcus Coyle, 1988 \\
\hline & Euagrus pristinus O. Pickard-Cambridge, 1899 \\
\hline Euctenizidae & Eucteniza hidalgo Bond \& Godwin, 2013 \\
\hline \multirow[t]{5}{*}{ Gnaphosidae } & Drassyllus conformans Chamberlin, 1936 \\
\hline & Drassyllus zimus Platnick \& Shadab, 1982 \\
\hline & Herpyllus cockerelli Banks, 1901 \\
\hline & Herpyllus perditus Banks, 1898 \\
\hline & Herpyllus perote Platnick \& Shadab, 1977 \\
\hline
\end{tabular}




\begin{tabular}{|c|c|}
\hline Familia & Género y/o especie \\
\hline & Herpyllus propinquus Keyserling, 1887 \\
\hline & Micaria deserticola Gertsch, 1933 \\
\hline & Micaria emertoni Gertsch, 1935 \\
\hline & Micaria longipes Emerton, 1890 \\
\hline & Tivodrassus ethophor Chamberlin \& Ivie, 1936 \\
\hline & Zelotes anglo Gertsch \& Riechert, 1976 \\
\hline & Zelotes lasalanus Chamberlin, 1928 \\
\hline & Zelotes moestus O. Pickard-Cambridge, 1898 \\
\hline \multirow[t]{4}{*}{ Hahniidae } & Hahnia nobilis Opell \& Beatty, 1976 \\
\hline & Neoantistea hidalgoensis Opell \& Beatty, 1976 \\
\hline & Neoantistea jacalana Gertsch, 1946 \\
\hline & Neoantistea lyrica Opell \& Beatty, 1976 \\
\hline Leptonetidae & Neoleptoneta brunnea Gertsch, 1974 \\
\hline \multirow[t]{5}{*}{ Linyphiidae } & Eulaira hidalgoana Gertsch \& Davis, 1937 \\
\hline & Frontinella pyramitela Walckenaer, 1841 \\
\hline & Linyphia nigrita F. O. Pickard-Cambridge, 1902 \\
\hline & Mermessus tepejicanus Gertsch \& Davis, 1937 \\
\hline & Toltecaria antricola Millidge, 1984 \\
\hline \multirow{19}{*}{ Lycosidae } & Allocosa apora Gertsch, 1934 \\
\hline & Allocosa mokiensis Gertsch, 1934 \\
\hline & Allocosa parva Banks, 1894 \\
\hline & Hogna tlaxcalana Gertsch \& Davis, 1940 \\
\hline & Lycosa sp. \\
\hline & Pardosa falcifera F. O. Pickard-Cambridge, 1902 \\
\hline & Pardosa flavipalpis F. O. Pickard-Cambridge, 1902 \\
\hline & Pardosa marialuisae Dondale \& Redner, 1984 \\
\hline & Pardosa mayana Dondale \& Redner, 1984 \\
\hline & Pardosa prolífica F. O. Pickard-Cambridge, 1902 \\
\hline & Pardosa sternalis Thorell, 1877 \\
\hline & Pardosa sura Chamberlin \& Ivie, 1941 \\
\hline & Pirata sedentarius Montgomery, 1904 \\
\hline & Schizocosa avida Walckenaer, 1837 \\
\hline & Schizocosa crassipalpata Roewer, 1951 \\
\hline & Schizocosa crassipes Walckenaer, 1837 \\
\hline & Schizocosa mimula Gertsch, 1934 \\
\hline & Schizocosa saltatrix Hentz, 1844 \\
\hline & Tigrosa helluo Walckenaer, 1837 \\
\hline \multirow[t]{5}{*}{ Nesticidae } & Eidmannella pallida Emerton, 1875 \\
\hline & Gaucelmus augustinus Keyserling, 1884 \\
\hline & Gaucelmus calidus Gertsch, 1971 \\
\hline & Nesticus hoffmanni Gertsch, 1971 \\
\hline & Nesticus sp. \\
\hline \multirow[t]{4}{*}{ Oxyopidae } & Oxyopes apollo Brady, 1964 \\
\hline & Oxyopes salticus Hentz, 1845 \\
\hline & Peucetia longipalpis F. O. Pickard-Cambridge, 1902 \\
\hline & Peucetia viridans Hentz, 1832 \\
\hline Philodromidae & Apollophanes punctipes O. Pickard-Cambridge, 1891 \\
\hline
\end{tabular}




\begin{tabular}{|c|c|}
\hline Familia & Género y/o especie \\
\hline Pholcidae & $\begin{array}{l}\text { Coryssocnemis iviei Gertsch, } 1971 \\
\text { Ixchela abernathyi Gertsch, } 1971 \\
\text { Ixchela huasteca Valdez-Mondragón, } 2013 \\
\text { Ixchela simoni O. Pickard-Cambridge, } 1898 \\
\text { Modisimus guerrerensis Gertsch \& Davis, } 1937 \\
\text { Pholcophora texana Gertsch, } 1935 \\
\text { Physocyclus fishi Gertsch, } 1971 \\
\text { Physocyclus franckei Valdez-Mondragon, } 2010 \\
\text { Psiolochorus reddelli Gertsch, } 1971 \\
\text { Psilochorus utahensis Chamberlin, } 1919\end{array}$ \\
\hline Phrurolithidae & Phrurolithus tepejicanus Gertsch \& Davis, 1940 \\
\hline Plectreuridae & Plectreurys paisana Gertsch, 1958 \\
\hline Salticidae & $\begin{array}{l}\text { Habronattus fallax Peckham \& Peckham, } 1909 \\
\text { Habronattus mexicanus Peckham \& Peckham, } 1896 \\
\text { Habronattus oregonesis Peckham \& Peckham, } 1888 \\
\text { Habronattus virgulatus Griswold, } 1987 \\
\text { Lyssomanes diversus Galiano, } 1980 \\
\text { Lyssomanes malinche Galiano, } 1980 \\
\text { Paraphidippus aurantius Lucas, } 1833 \\
\text { Pelegrina furcata F. O. Pickard-Cambridge, } 1901 \\
\text { Pelegrina neoleonis Maddison, } 1996 \\
\text { Phidippus amans Edwards, } 2004 \\
\text { Phidippus arizonensis Peckham \& Peckham, } 1883 \\
\text { Phidippus carneus Peckham \& Peckham, } 1896 \\
\text { Phidippus cerberus Edwards, } 2004 \\
\text { Phidippus georgii Peckham \& Peckham, } 1896 \\
\text { Sassacus lirios Richman, } 2008 \\
\text { Sassacus vitis Cockerell, } 1894\end{array}$ \\
\hline Scytodidae & $\begin{array}{l}\text { Scytodes intricata Banks, } 1909 \\
\text { Scytodes itzana Chamberlin \& Ivie, } 1938 \\
\text { Scytodes opoxtli Rheims, Brescovit \& Durán-Barrón, } 2007\end{array}$ \\
\hline Segestriidae & Ariadna pilífera O. Pickard-Cambridge, 1898 \\
\hline Selenopidae & Selenops mexicanus Keyserling, 1880 \\
\hline Sicariidae & $\begin{array}{l}\text { Loxosceles jaca Gertsch \& Ennik, } 1983 \\
\text { Loxosceles nahuana Gertsch, } 1958 \\
\text { Loxosceles tenango Gertsch, } 1973 \\
\text { Loxosceles tolantongo Navarro-Rodríguez \& Valdez-Mondragón, } 2020\end{array}$ \\
\hline Tetrablemmidae & Caraimatta sbordonii Brignoli, 1972 \\
\hline Tetragnathidae & $\begin{array}{l}\text { Cyrtognatha paradoxa Dimitrov \& Hormiga, } 2009 \\
\text { Glenognatha spherella Chamberlin \& Ivie, } 1936\end{array}$ \\
\hline Theraphosidae & Bonnetina aviae Estrada-Alvarez \& Locht, 2011 \\
\hline Theridiidae & $\begin{array}{l}\text { Anelosimus baeza Agnarsson, } 2006 \\
\text { Anelosimus octavius Agnarsson, } 2006 \\
\text { Anelosimus oritoyacu Agnarsson, } 2006 \\
\text { Anelosimus studiosus Hentz, } 1850 \\
\text { Ariamnes mexicanus Exline \& Levi, } 1962 \\
\text { Asagena americana Emerton, } 1882\end{array}$ \\
\hline
\end{tabular}




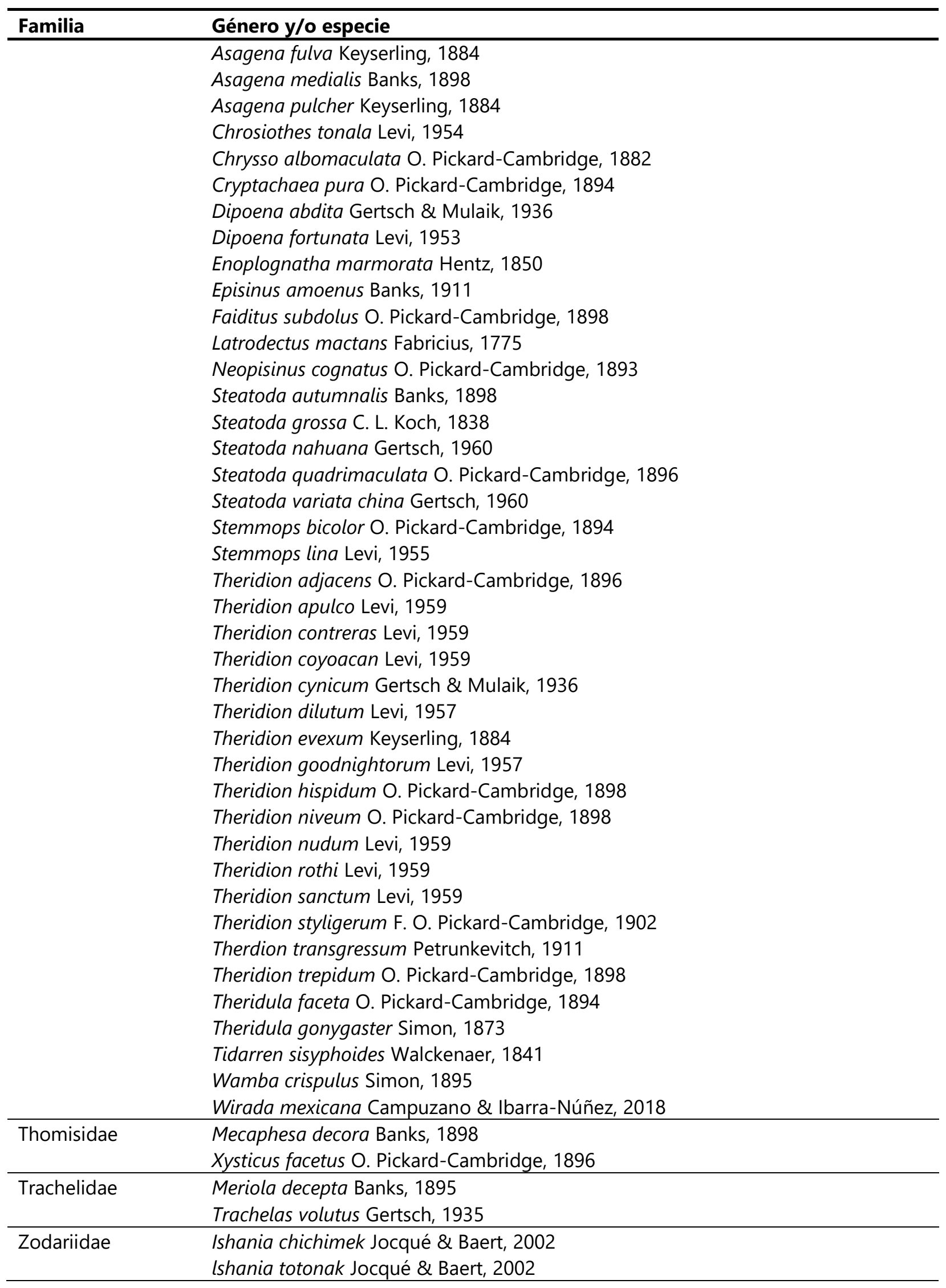


Familia

Zoropsidae

\section{Género y/o especie}

Zorocrates apulco Platnick \& Ubick, 2007

Zorocrates fuscus Simon, 1888

Zorocrates sotano Platnick \& Ubick, 2007

Zorocrates unicolor Banks, 1901

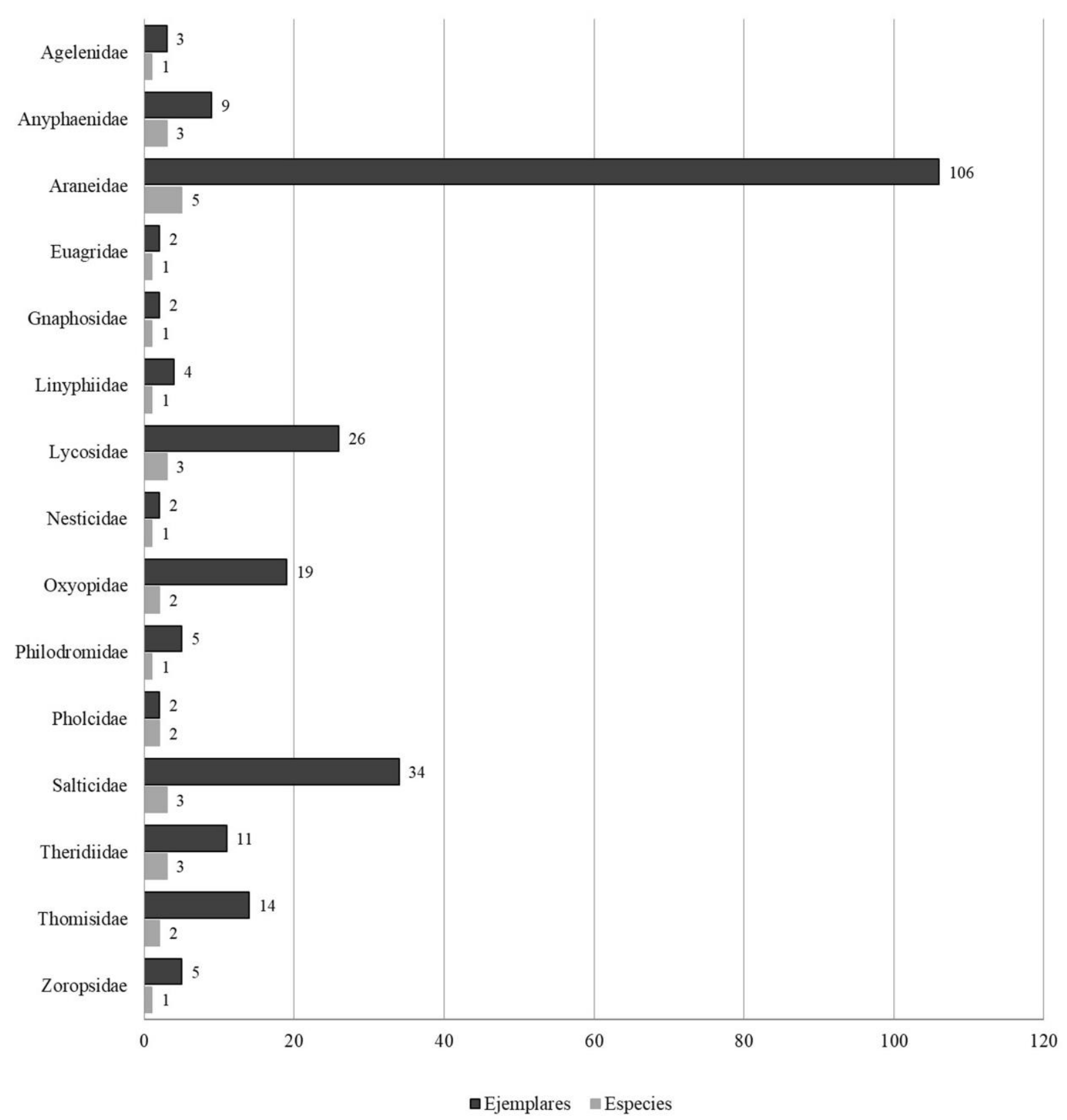

Figura 1. Número de ejemplares y especies por familia depositadas en la CAEM. 


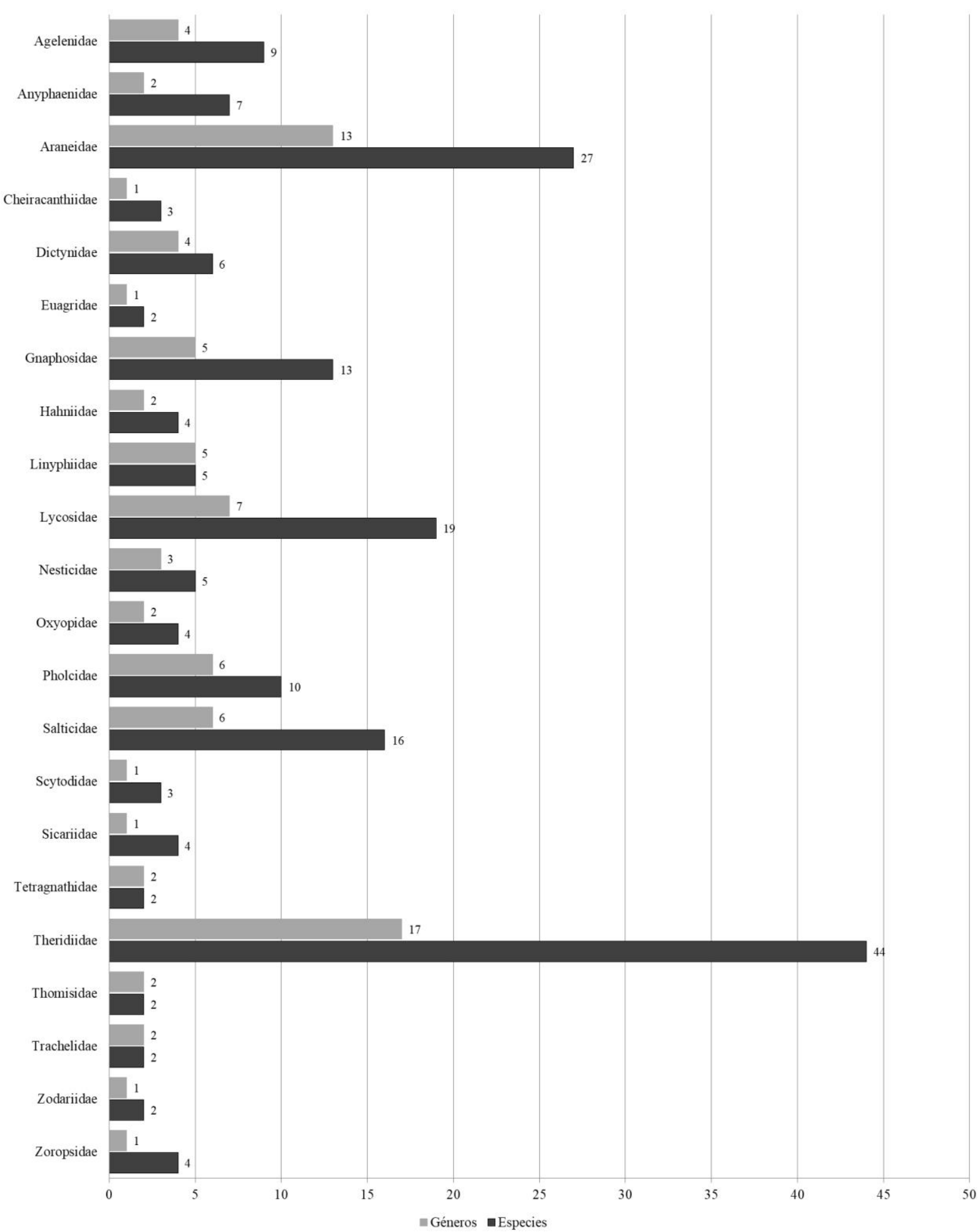

Figura 2. Número de géneros y especies por familia presentes en el estado de Hidalgo. La figura no incluye a las familias que solo están representadas por un género y una especie (Clubionidae, Corinnidae, Euctenizidae, Leptonetidae, Philodromidae, Phrurolithidae, Plectreuridae, Segestriidae, Selenopidae, Tetrablemmidae y Theraphosidae). 


\section{Nuevos Registros}

Familia Anyphaenidae Bertkau, 1878

\section{Anyphaena cf. hespar Platnick, 1974. Figs. 3-4.}

Material examinado: México, Hidalgo, Tepeji del Río de Ocampo, Santiago Tlautla. 2 $\delta^{\lambda}$ (CAEM/ARA0000133 y CAEM/ARA0000135), 21/09/2007. 2,145 m s.n.m. 1957'42.65" N, 99²2'02.21" O. Bosque de galería. Rodríguez, G. col. Distribución: Estados Unidos de América y México (World Spider Catalog, 2021). Registros previos en México: Chihuahua (Platnick \& Lau, 1975). Primer registro para Hidalgo.

\section{Anyphaena pacifica Banks, 1896. Figs. 5-7.}

Material examinado: México, Hidalgo, Tepeji del Río de Ocampo, Santiago Tlautla. 10 (CAEM/ARA0000154), 21/09/2007. 2,145 m s.n.m. 1957'42.65" N, 99²2'02.21" O. Bosque de galería. Pérez, A. col. Distribución: Canadá y Estados Unidos de América (World Spider Catalog, 2021). Primer registro para México.

\section{Familia Araneidae Clerck, 1757}

Eustala rosae Chamberlin \& Ivie, 1935. Figs. 8-9.

Material examinado: México, Hidalgo, Tepeji del Río de Ocampo: Santiago Tlautla. $2 q q$ (CAEM/ARA0000143), 21/09/2007. 2,145 m s.n.m. 1957'42.65" N, 99²2'02.21" O. Bosque de galería. Pérez, A. col. El Zapote. 5우 (CAEM/ARA0000144, CAEM/ARA0000146 y CAEM/ARA0000234), 26/10/2007. 2,255 m s.n.m. 1957'44.98' N, 99²4'36.72" O. Matorral xerófilo. Pérez, A. y Rodríguez, G. cols. Distribución: Estados Unidos de América y México (World Spider Catalog, 2021). Registros previos en México: Baja California y Chihuahua (Levi, 1977). Primer registro para Hidalgo.

\section{Familia Oxyopidae Thorell, 1870}

\section{Oxyopes salticus Hentz, 1845. Figs.10-11.}

Material examinado: México, Hidalgo, Tepeji del Río de Ocampo, Represa Cerro de la Virgen. 1 q (CAEM/ARA0000882), 26/10/2007. 2,252 m s.n.m. 1958'42"N, 99²5'07"O. Matorral xerófilo. Rodríguez, G. col. Distribución: desde Estados Unidos de América hasta el norte de Argentina y Chile (World Spider Catalog, 2021). Registros previos en México: Campeche, Chiapas, Chihuahua, Colima, Durango, Guerrero, Jalisco, Michoacán, Morelos, Nayarit, Nuevo León, Oaxaca, San Luis Potosí, Veracruz, Tamaulipas y Yucatán (Brady, 1975). Primer registro para Hidalgo.

\section{Familia Pholcidae C. L. Koch, 1850}

Ixchela abernathyi Gertsch, 1971. Figs. 12-13.

Material examinado: México, Hidalgo, Tepeji del Río de Ocampo, Santiago Tlautla. $1 \delta^{\lambda}$ (CAEM/ARA0000254), 21/09/2007. 2,145 m s.n.m. 1957'42.65" N, 99²2'02.21" O. Bosque de galería. Pérez, A. col. Distribución: México (World Spider Catalog, 2021). Registros previos en México: Coahuila, Nuevo León, San Luis Potosí y Tamaulipas (Valdez-Mondragón, 2013). Primer registro para Hidalgo. 


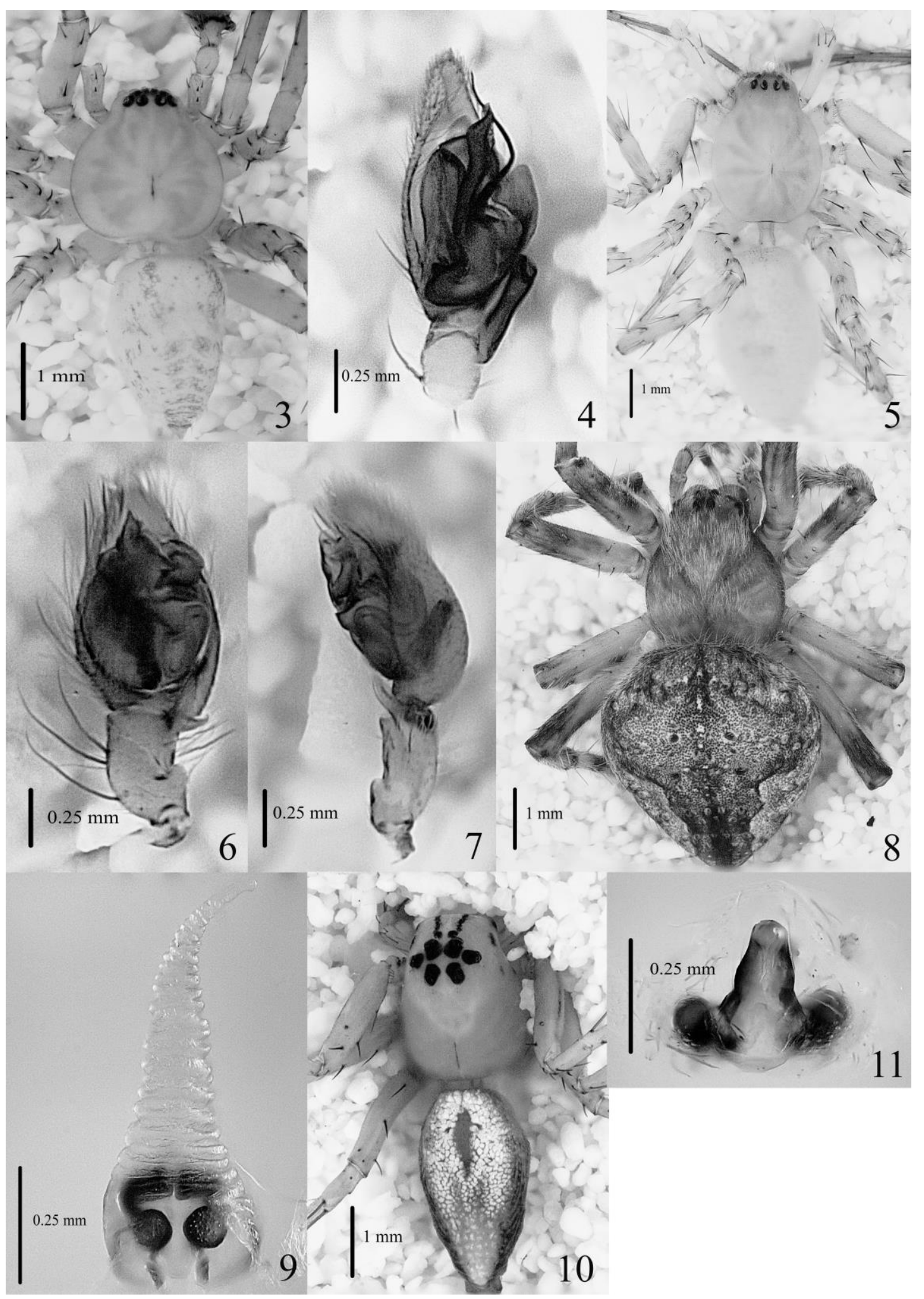

Figuras 3-11. Anyphaena cf. hespar 3) Macho en vista dorsal, 4) Pedipalpo en vista ventral; Anyphaena pacifica, 5) Macho en vista dorsal, 6) Pedipalpo en vista ventral, 7) Pedipalpo en vista retrolateral; Eustala rosae, 8) Hembra en vista dorsal, 9) Epigineo en vista ventral; Oxyopes salticus, 10) Hembra en vista dorsal, 11) Epigineo en vista ventral. 


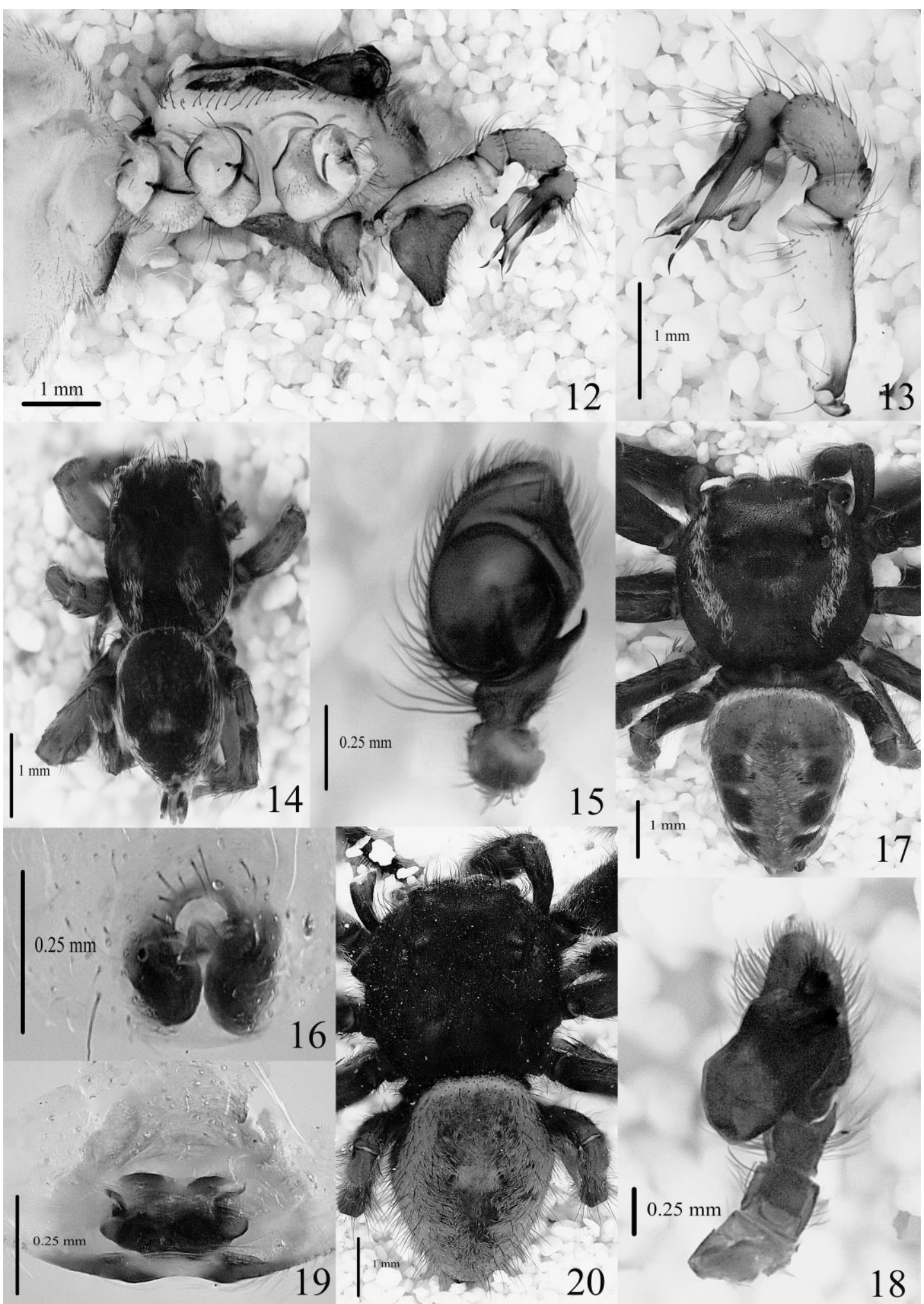

Figuras 12-20. Ixchela abernathyi, 12) Macho en vista lateral, 13) Pedipalpo en vista retrolateral; Habronattus oregonensis, 14) Macho en vista dorsal, 15) Pedipalpo en vista ventral, 16) Epigineo en vista ventral; Paraphidippus aurantius, 17) Macho en vista dorsal, 18) Pedipalpo en vista ventral, 19) Epigineo en vista dorsal; Phidippus carneus, 20) Macho en vista dorsal. 


\section{Familia Salticidae Blackwall, 1841}

\section{Habronattus oregonesis Peckham \& Peckham, 1888. Figs. 14-16.}

Material examinado: México, Hidalgo, Tepeji del Río de Ocampo, Represa Cerro de la Virgen. $2 q q$ (CAEM/ARA0000216) y 10 (CAEM/ARA0000245), 20/09/2007. 2,252 m s.n.m. 1958'42" N, 99²5'07" O. Matorral xerófilo. Rodríguez, G. col. Distribución: Norteamérica (World Spider Catalog, 2021). Registros previos en México: Baja California (Jiménez \& Palacios-Cardiel, 2012). Primer registro para Hidalgo.

\section{Paraphidippus aurantius Lucas, 1833. Figs. 17-19.}

Material examinado: México, Hidalgo, Tepeji del Río de Ocampo, El Zapote. 4ðð (CAEM/ARA0000251, CAEM/ARA0000997, CAEM/ARA0000992 y CAEM/ARA0000993) y $5 q q$ (CAEM/ARA0000243， CAEM/ARA0000249, CAEM/ARA0000994, CAEM/ARA0000995 y CAEM/ARA0000996), 26/10/2007. 2,240 m s.n.m. 1958'2.3" N, 99²4'50.3" O. Matorral xerófilo. Pérez, A. col. Distribución: de Estados Unidos de América a Panamá, hasta las Antillas Mayores (World Spider Catalog, 2021). Registros previos en México: Ciudad de México, Guerrero, Nayarit (Pickard-Cambridge, 1901), Estado de México (Jiménez-Jiménez, 1989), Michoacán (MaldonadoCarrizales \& Ponce-Saavedra, 2017). Primer registro para Hidalgo.

\section{Phidippus carneus Peckham \& Peckham, 1896. Figs. 20-22.}

Material examinado: México, Hidalgo, Tepeji del Río de Ocampo, Represa Cerro de la Virgen. $3 \hat{\jmath} \widehat{\jmath}$ (CAEM/ARA0000242, CAEM/ARA0000244 y CAEM/ARA0000247), 26/10/2007. 2,252 m s.n.m. $19^{\circ} 58^{\prime} 42^{\prime \prime}$ N, 99²5'07" O. Matorral xerófilo. Rodríguez, G. y Pérez, A. cols. Distribución: Estados Unidos de América y México (World Spider Catalog, 2021). Registros previos en México: Aguascalientes, Baja California, Chihuahua, Coahuila, Guanajuato, Jalisco, San Luis Potosí, Sonora y Zacatecas (Edwards, 2004). Primer registro para Hidalgo.

\section{Familia Theridiidae Sundevall, 1833}

\section{Steatoda grossa C. L. Koch, 1838. Figs. 23-24.}

Material examinado: México, Hidalgo, Tepeji del Río de Ocampo, Represa Cerro de la Virgen. $1 q$ (CAEM/ARA0000744), 20/09/2007. 2,252 m s.n.m. 1958'42" N, 99²5'07" O. Matorral xerófilo. Rodríguez, G. y Pérez, A. cols. Distribución: El Cáucaso, Rusia, Turquía (Europa hasta el Lejano Oriente), Asia Central, China, Corea y Japón. Introducida en América del Norte y Hawái, Chile, Ecuador y Perú en Sudamérica, Argelia y Macaronésica en África (World Spider Catalog, 2021). Registros previos en México: Baja California, Chihuahua, Ciudad de México, Estado de México, Michoacán, Morelos, Puebla, Sonora, Veracruz, Zacatecas (Levi, 1957), Durango (Levi, 1962), Chiapas, Colima, Durango, Guerrero, Jalisco, Nayarit, Oaxaca y Sinaloa (Levi, 1967). Primer registro para Hidalgo.

Theridion niveum O. Pickard-Cambridge, 1898. Figs. 25-27.

Material examinado: México, Hidalgo, Tepeji del Río de Ocampo, Santiago Tlautla. $2 q q$ (CAEM/ARA0000749 y CAEM/ARA0000991), 21/09/2007. 2,145 m s.n.m. 1957'42.65" N, 99²2'02.21" O. Bosque de Galería. Morán, D. col. Distribución: México (World Spider Catalog, 2021). Registros previos en México: Tabasco (Pickard-Cambridge, 1902), Tamaulipas (Chickering, 
1937), Chiapas, Guerrero, Oaxaca, San Luis Potosí, Veracruz (Levi, 1959). Primer registro para Hidalgo.

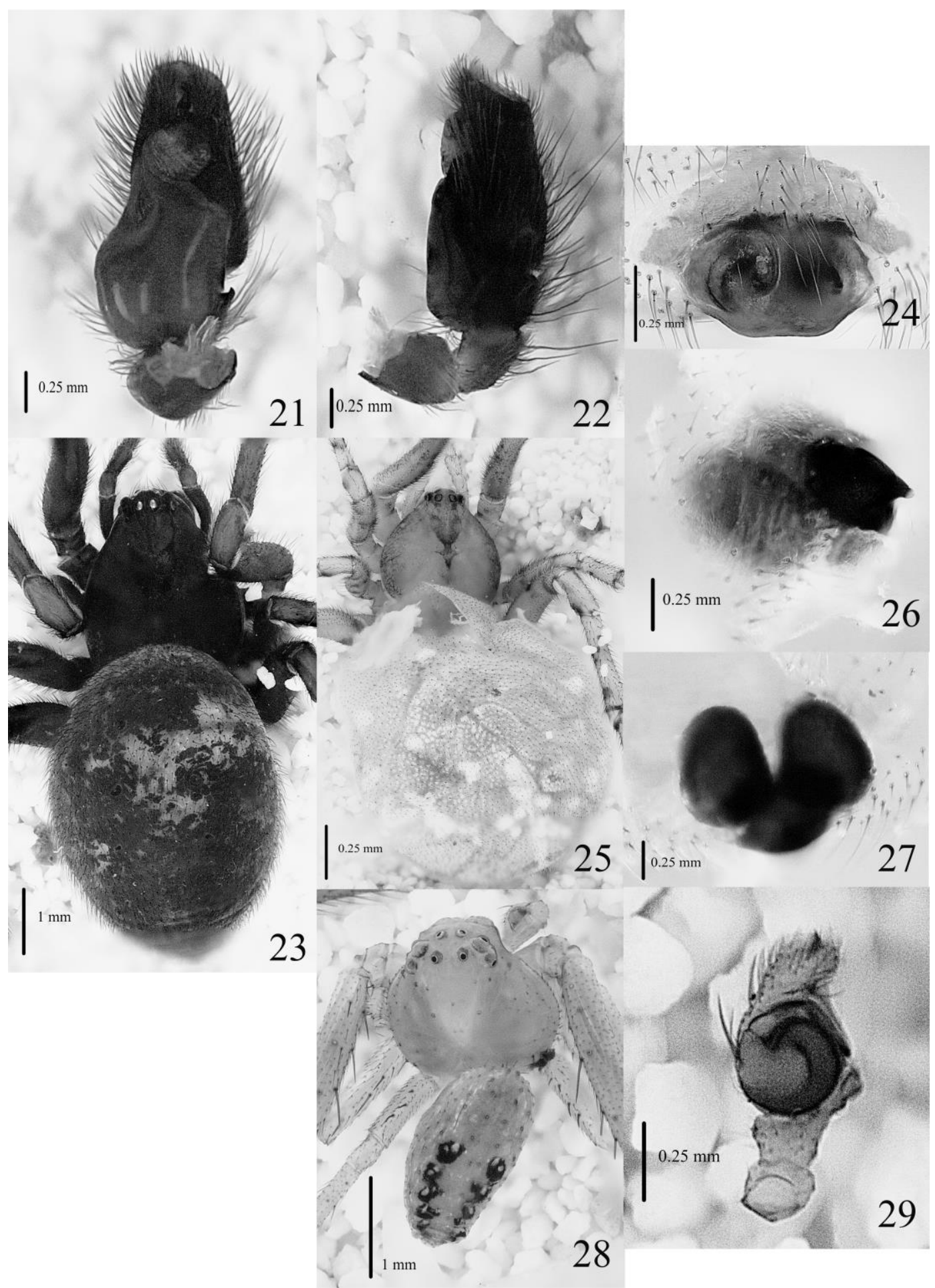

Figuras 21-29. Phidippus carneus, 21) Pedipalpo en vista ventral, 22) Pedipalpo en vista retrolateral; Steatoda grossa, 23) Hembra en vista dorsal, 24) Epigineo en vista ventral; Theridion niveum, 25) Hembra en vista dorsal, 26) Epigineo en vista lateral, 27) Epigineo en vista dorsal; Mecaphesa decora, 28) Macho en vista dorsal, 29) Pedipalpo en vista ventral. 


\section{Familia Thomisidae Sundevall, 1833 \\ Mecaphesa decora Banks, 1898. Figs. 28-29.}

Material examinado: México, Hidalgo, Tepeji del Río de Ocampo, El Zapote. 20̂̉ (CAEM/ARA0000153 y CAEM/ARA0000155), 20/09/2007. 2,255 m s.n.m. 1957'44.98" N, 99²4'36.72" O. Matorral xerófilo. Rodríguez, G. y Pérez, A. cols. Distribución: Guatemala y México (World Spider Catalog, 2021). Registros previos en México: Baja California (Banks, 1898), Ciudad de México, Jalisco, Veracruz (Gertsch, 1939) y Estado de México (Durán-Barrón, 2004). Primer registro para Hidalgo.

\section{DISCUSIÓN Y CONCLUSIONES}

Jiménez (1996) situó al estado de Hidalgo en el décimo quinto lugar de diversidad de arañas en México. Con el presente trabajo Hidalgo ocupa el octavo puesto, ya que pasa de tener 125 a 207 especies. Esto indica que el conocimiento de la diversidad de arañas presente en el estado de Hidalgo se incrementó en un 61.38\%. Las 207 especies reportadas para el estado representan el $8.66 \%$ de las especies registradas para México respecto al número de especies indicadas por Durán-Barrón et al. (2016), mientras que a nivel mundial los datos corresponden al $25.78 \%$ de las familias, el $2.40 \%$ de los géneros y apenas el $0.42 \%$ de la riqueza de especies en el mundo, de acuerdo con los datos del World Spider Catalog (2021). Nuestros resultados indican que las arañas representan el tercer grupo zoológico más diverso en Hidalgo, superado únicamente por los insectos (1,025especies) y las aves (501 especies) (CONABIO y SEMARNAT, 2020).

La distribución de A. pacifica únicamente era conocida en el oeste de los Estados Unidos, desde el sur de California hasta Columbia Británica, tierra adentro hacia Nuevo México y Alberta, habitando en casas, bajo la hojarasca y bajo rocas (Dondale \& Redner, 1982). Con el nuevo registro de esta especie en México se amplía su distribución hasta la ZTM, en lo que geográficamente corresponde a la Provincia del EVT. Con el nuevo registro de $A$. cf. hespar se amplía la distribución de esta especie desde la región norteña del Desierto Chihuahuense (Platnick \& Lau, 1975) hasta la ZTM en la Provincia del EVT, por lo que es probable que se distribuya a lo largo del Altiplano Mexicano. Eustala rosae fue reportada en bosques de montaña el noroeste de los Estados Unidos, en matorrales de la región norteña del Desierto Chihuahuense (Levi, 1977) y en los oasis de Baja California (Jiménez et al., 2015). Con nuestros resultados se amplía la distribución de esta especie hasta la ZTM en el EVT. Asimismo, se amplía la distribución de $H$. oregonensis hasta la ZTM en la provincia del EVT a más de 2,000 metros de altitud en matorral xerófilo; anteriormente solo era reportada a lo largo de las costas del Pacifico, desde Columbia Británica hasta Baja California, donde fue reportada en bosque de Quercus sp. a 1,775 m s.n.m. (Griswold, 1987; Jiménez \& Palacios-Cardiel, 2012). Valdez-Mondragón (2013) reporta a I. abernathyi en zonas kársticas con bosques de pino y encino en la región norte del Altiplano Mexicano, parte de la Sierra Madre Oriental y Tamaulipas, con el nuevo registro se amplía la distribución de esta especie hacia la ZTM en el EVT en bosque de galería. La especie $P$. aurantius estaba reportada en los bosques templados del EVT (Maldonado-Carrizales \& Ponce-Saavedra, 2017; Jiménez-Jiménez, 1989; Hoffmann, 1976), por lo que con el nuevo registro únicamente se amplía la distribución de esta especie en dicha provincia en la ZTM en matorral xerófilo. Phidippus carneus se registra desde suroeste de los Estados Unidos hasta el centro de México, siempre recolectada en hábitats xéricos en un rango 
altitudinal que va desde los 128 hasta los 2,161 metros (Edwards, 2004), con el presente trabajo se amplía la distribución de esta especie en la ZTM en el EVT. Mecaphesa decora es reportada en la región de Baja California, en algunos estados del EVT y en las costas del Golfo de México (Hoffmann, 1976; Durán-Barrón, 2004), con el nuevo registro se amplía la distribución de esta especie en el EVT. Las especies O. salticus, S. grossa y T. niveum se distribuyen ampliamente por el país (Hoffmann, 1976; Brady, 1975) y con los nuevos registros presentados se amplía la distribución de estas especies en la Provincia del EVT en la ZTM.

En cuanto a las especies Apollophanes punctipes O. Pickard-Cambridge, 1891 (Philodromidae), Eratigena mexicana Roth, 1968, Eratigena tlaxcala Roth, 1968 (Agelenidae) y Oxyopes apollo Brady, 1964 (Oxiopidae), fueron citadas para Hidalgo en los trabajos de Dondale y Redner (1975), Roth (1968) y Brady (1975), respectivamente. Sin embargo, aunque Hoffmann (1976) consultó estos trabajos, omitió por error a las especies antes indicadas. Gertsch (1958), por su parte, indicó que es probable que la especie Plectreurys paisana Gertsch, 1958 se distribuya en Hidalgo debido a que revisó ejemplares inmaduros recolectados en este estado y que posiblemente pertenezcan a esta especie pues su distribución es cercana al estado de Hidalgo, por lo tanto, decidimos incluirla en el listado. La especie Pardosa sierra Banks, 1898 es omitida del listado de especies a pesar de que Hoffmann (1976) la cita de Hidalgo, pues Correa-Ramírez et al. (2010) en los estudios filogeográficos de esta especie demostraron que $P$. sierra es endémica de la Península de Baja California y erróneamente fue identificada como tal para el interior de la República Mexicana. Levi (2004) no cita textualmente a A. trifasciata para el estado de Hidalgo, sin embargo, en el mapa de distribución de la especie aparece un punto para la entidad, por lo cual se decidió añadir el registro de la especie. Aunado a esto, en la CAEM se encuentran depositados dos especímenes de esta especie provenientes de Hidalgo, lo cual corrobora la presencia de $A$. trifasciata en el estado.

El conocimiento de la biodiversidad, importancia cultural, económica y ecológica de las arañas aún es limitado a nivel nacional, por lo que es necesario impulsar la generación de conocimiento de este grupo en diferentes perspectivas para que se reconozca la importancia de las arañas del país y lleguen a ser consideradas en la toma de decisiones entorno a la conservación y el aprovechamiento de los recursos naturales. Además, es pertinente implementar acciones concretas de divulgación y educación ambiental acerca de la biología e importancia de estos organismos, de tal manera que se contribuya a desechar las creencias erróneas y reducir el temor exagerado y generalizado hacia todas las especies (Ibarra-Núñez, 2013).

Las colecciones científicas son de gran importancia para todas las instituciones que se dedican a la investigación y divulgación científica. Estas representan una valiosa herramienta que permite generar, validar o perfeccionar el conocimiento científico (Cristín \& Perrilliat, 2011). Además, forman parte del patrimonio natural de un país o región, en ellas se preservan ejemplares e información valiosa que se encuentra disponible para la comunidad científica (Cotterill, 1997). Es necesario promover el desarrollo de las colecciones científicas en nuestro país, disminuyendo las carencias que estas puedan presentar y mediante la recolecta y depósito de organismos. Por último, en las colecciones de arácnidos del país se encuentra depositado material que permanece 
sin ser examinado, por lo que es pertinente comenzar una revisión del material con el fin de incrementar el conocimiento de la biodiversidad de arañas de México.

AgradeCimientos. Los autores agradecen a la Dra. Petra Sánchez Nava y al Dr. Felipe de Jesús Rodríguez Romero por permitirnos trabajar en el Laboratorio de Sistemas Biosustentables de la Facultad de Ciencias de la Universidad Autónoma del Estado de México (UAEMéx). A la M. en C. Dariana R. Guerrero-Fuentes por la corroboración de las especies de la familia Salticidae. Al M. en C. Giovany Arturo González-Desales por instruirnos en la técnica de montaje de imágenes en Adobe Photoshop CS6. A los Biólogos Regina Valdovinos Uribe y Daniel Gachuz Bracamontes por la revisión del resumen en inglés. A los revisores anónimos por sus atinados comentarios para mejorar el manuscrito.

\section{LITERATURA CITADA}

Banks, N. (1898) Arachnida from Baja California and other parts of Mexico. Proceedings of the California Academy of Sciences, 3 (1), 205-309.

Brady, A. R. (1975) The lynx spider genus Oxyopes in Mexico and Central America (Araneae: Oxyopidae). Psyche, Cambridge, 82 (2), 189-243. https://doi.org/10.1155/1975/24938

Campuzano, E. F., Ibarra-Núñez, G. (2018) A new species of the spider genus Wirada (Araneae, Theridiidae) from Mexico, with taxonomic notes on the genus and a key to the species. Zootaxa, 4457 (3), 495-500. https://doi.org/10.11646/zootaxa.4457.3.13

Chamé-Vázquez, D., Ibarra-Núñez, G. (2019) A new species of Phonotimpus Gertsch \& Davis, 1940 (Araneae: Phrurolithidae) from Mexico. Zootaxa, 4545 (1), 146-150. https://doi.org/10.11646/zootaxa.4545.1.10

Chamé-Vázquez, D., Ibarra-Núñez, G., Jiménez, M. L. (2018) Redescription of Phonotimpus separatus Gertsch \& Davis, 1940 (Araneae: Phrurolithidae) and description of two new species of Phonotimpus from Mexico. Zootaxa, 4407 (2), 213-228. https://doi.org/10.11646/zootaxa.4407.2.3

Chickering, A. M. (1937) Notes and studies on Arachnida. III. Arachnida from the San Carlos Mountains. Pp. 271-283. In: The Geology and Biology of the San Carlos Mountains, Tamaulipas, Mexico. University of Michigan Press, USA.

Coddington, J. A., Levi, H. W. (1991) Systematics and evolution of spiders. Annual Review of Ecology and Systematics, 22, 565-592.

Comisión Nacional para el Conocimiento y Uso de la Biodiversidad (CONABIO), Secretaría de Medio Ambiente y Recursos Naturales del Estado de Hidalgo (SEMARTH) (2020) Estrategia para la Conservación y el Uso Sustentable de la Biodiversidad del Estado de Hidalgo (ECUSBEH). CONABIO/SEMARNARTH, México, 206 pp.

Correa-Ramírez, M. M., Jiménez, M. L., García-De León, F. J. (2010) Testing species boundaries in Pardosa sierra (Araneae: Lycosidae) using female morphology and COI mtDNA. Journal of Arachnology, 38, 538-554. 
Cotterill, F. P. D. (1997) The second Alexandrian tragedy and the fundamental relationship between biological collections and scientific knowledge. Pp. 227-241. In: J. R. Nudds, C. W. Pettitt (Eds.). The value and valuations of natural science collections: proceedings of the international conference. Manchester, Reino Unido, 1995, The Geological Society.

Craig, C. L. (2003) Spiderwebs and Silk. Tracing Evolution from Molecules to Genes to Phenotypes. Oxford University Press, New York, 230 pp.

Cristín, A., Perrilliat, M. C. (2011) Las colecciones científicas y la protección del patrimonio paleontológico. Boletín de la Sociedad Geológica Mexicana, 63, 421-427.

Desales-Lara, M. A. (2014) Araneofauna (Arachnida: Araneae) del Estado de México, México. Acta Zoológica Mexicana (nueva serie), 30 (2), 298-320.

Desales-Lara, M. A., Jiménez, M. L., Corcuera, P. (2018) Nuevos registros de arañas (Arachnida: Araneae) para México y listado actualizado de la araneofauna del estado de Coahuila. Acta Zoológica Mexicana (nueva serie), 34 (1), 50-63. https://doi.org/10.21829/azm.2018.3411183

Dondale, C. D., Redner, J. H. (1975) Revision of the spider genus Apollophanes (Araneida: Thomisidae). The Canadian Entomologist, 107 (11), 1175-1192. https://doi.org/10.4039/Ent1071175-11

Dondale, C. D., Redner, J. H. (1982) The insects and arachnids of Canada, Part 9. The sac spiders of Canada and Alaska, Araneae: Clubionidae and Anyphaenidae. Research Branch Agriculture Canada Publication, 1724, 1-194.

Durán-Barrón, C. G. (2004) Diversidad de arañas (Arachnida, Araneae) asociadas a viviendas de la Ciudad de México. Tesis de Maestría, Instituto de Biología, Universidad Nacional Autónoma de México, México, 229 pp.

Durán-Barrón, C. G., Montiel-Parra, G., Valdez-Mondragón, A., Villegas-Guzmán, G. A., Paredes-León, R., Pérez, T. M. (2016) Arácnidos (Arachnida). Pp. 229-238. In: La biodiversidad en la Ciudad de México. Vol. II. CONABIO/SEDEMA, México.

Edwards, G. B. (2004) Revision of the jumping spiders of the genus Phidippus (Araneae: Salticidae). Occasional Papers of the Florida State Collection of Arthropods, 11, 1-156.

Foelix, R. F. (2011) Biology of spiders, Third edition. Oxford University Press, New York, 419 pp.

García-Villafuerte, M. A. (2009) La araneofauna (Araneae) reciente y fósil de Chiapas, México. Revista Mexicana de Biodiversidad, 80, 633-646. http://dx.doi.org/10.22201/ib.20078706e.2009.003.160

Gertsch, W. J. (1939) A revision of the typical crab spiders (Misumeninae) of America north of Mexico. Bulletin of the American Museum of Natural History, 76, 277-442.

Gertsch, W. J. (1958) The spider family Plectreuridae. American Museum Novitates, 1920, 1-53.

Gómez-Rodríguez, J. F., Montaño, H., Ibarra-Núñez, G., Salazar-Olivo, C. A. (2014) Arácnidos (excepto ácaros) de Tamaulipas: listado actualizado y algunos registros nuevos. Pp: 51-74. In: A. Correa-Sandoval, J. V. Horta, J. García-Jiménez, L. Barrientos (Eds.). Biodiversidad Tamaulipeca. Vol. 2. No. 2. Tecnológico Nacional de México - Instituto Tecnológico de Ciudad Victoria, Tamaulipas, México.

Griswold, C. E. (1987) A revision of the jumping spider genus Habronattus F. O. P.-Cambridge (Araneae; Salticidae), with phenetic and cladistics analyses. The University of California Publications in Entomology, 107, 1-344. 
Guerrero-Fuentes, D. R., Desales-Lara, M. A. (2020) Nuevos registros de arañas (Araneae) del Estado de México, México. Revista Ibérica de Aracnología, 36, 147-149.

Guerrero-Fuentes, D. R., Francke, O. F. (2019) Taxonomic revision of Anicius Chamberlin, 1925 (Araneae: Salticidae), with five new species of jumping spiders from Mexico. Zootaxa, 4638 (4), 485-506. https://doi.org/10.11646/zootaxa.4638.4.2

Hoffmann, A. (1976) Relación bibliográfica preliminar de las arañas de México (Arachnida: Araneae). Publicaciones Especiales No. 3, Instituto de Biología, Universidad Nacional Autónoma de México, México, 117 pp.

Hoffmann, A. (1993) El maravilloso mundo de los arácnidos. Fondo de Cultura Económica, México, $166 \mathrm{pp}$.

Ibarra Núñez, G. (2011) Arañas (Chelicerata: Arachnida: Araneae). Pp. 269-276. In: La biodiversidad en Veracruz: Estudio de Estado. Vol. 2. Comisión Nacional para el Conocimiento y Uso de la Biodiversidad (CONABIO), Gobierno del Estado de Veracruz, Universidad Veracruzana e Instituto de Ecología, A.C. México.

Ibarra-Núñez, G. (2013) Diversidad de las Arañas (Arachnida: Araneae). Pp. 191-196. In: La biodiversidad en Chiapas: Estudio de Estado. Comisión Nacional para el Conocimiento y Uso de la Biodiversidad (CONABIO) y Gobierno del Estado de Chiapas, México.

INEGI (2009) Prontuario de información geográfica municipal de los Estado Unidos Mexicanos, Tepeji del Río de Ocampo, Hidalgo. Instituto Nacional de Estadística y Geografía. Disponible en: http://www3.inegi.org.mx/contenidos/app/mexicocifras/datos_geograficos/13/13063.pdf (consultado 16 julio 2020).

Jiménez-Jiménez, M. L. (1989) Las arañas Araneomorphae de San Francisco Oxtotilpan Estado de México. Tesis Doctoral, Facultad de Ciencias, Universidad Nacional Autónoma de México, México, 303 pp.

Jiménez, M. L. (1996) Araneae. Pp. 83-101. In: J. Llorente, A. N. García-Aldrete, E. González (Eds.). Biodiversidad, taxonomía y biogeografía de artrópodos de México: hacia una síntesis de su conocimiento. Universidad Nacional Autónoma de México, México, D.F.

Jiménez, M. L., Palacios-Cardiel, C. (2012) Registros nuevos de arañas para el estado de Baja California, México. Acta Zoológica Mexicana (nueva serie), 28, 649-658.

Jiménez, M. L., Nieto-Castañeda, I. G., Correa-Ramírez, M. M., Palacios-Cardiel, C. (2015) Las arañas de los oasis de la región meridional de la península de Baja California, México. Revista Mexicana de Biodiversidad, 86, 319-331. http://dx.doi.org/10.1016/j.rmb.2015.04.028

Jiménez, M. L., Palacios-Cardiel, C., Chávez-Lara, I. D. (2020) Spiders (Arachnida: Araneae) of the Medanos of Samalayuca, Chihuahua and new records of species for Mexico. Acta Zoológica Mexicana (nueva serie), 36, 1-23.

https://doi.org/10.21829/azm.2020.3612205

Levi, H. W. (1957) The spider genera Crustulina and Steatoda in North America, Central America, and the West Indies (Araneae, Theridiidae). Bulletin of the Museum of Comparative Zoology, $117,367-424$. 
Levi, H. W. (1959) The spider genera Achaearanea, Theridion and Sphyrotinus from Mexico, Central America and the West Indies (Araneae, Theridiidae). Bulletin of the Museum of Comparative Zoology, 121, 57-163.

Levi, H. W. (1962) The spider genera Steatoda and Enoplognatha in America (Araneae, Theridiidae). Psyche, Cambridge, 69 (1), 11-36. https://doi.org/10.1155/1962/42957

Levi, H. W. (1967) Cosmopolitan and pantropical species of theridiid spiders (Araneae: Theridiidae). Pacific Insects, 9, 175-186.

Levi, H. W. (1977) The American orb-weaver genera Cyclosa, Metazygia and Eustala north of Mexico (Araneae, Araneidae). Bulletin of the Museum of Comparative Zoology, 148, 61-127.

Levi, H. W. (2004) Comments and new records for the American genera Gea and Argiope with the description of new species (Araneae: Araneidae). Bulletin of the Museum of Comparative Zoology, 158, 47-65.

Maldonado-Carrizales, J., Ponce-Saavedra, J. (2017) Arañas Saltarinas (Araneae: Salticidae) en dos sitios contrastantes en grado de antropización en Morelia Michoacán, México. Entomología mexicana, 4, 597-603.

Maya-Morales, J., Jiménez, M. L. (2017) Two new species of Melpomene from Mexico and description of the female of Melpomene elegans (Araneae: Agelenidae). Revista Mexicana de Biodiversidad, 88 (3), 579-586.

http://dx.doi.org/10.1016/j.rmb.2017.07.013

Mendoza, J. I., Francke, O. F. (2018) Five new cave-dwelling species of Hemirrhagus Simon 1903 (Araneae, Theraphosidae, Theraphosinae), with notes on the generic distribution and novel morphological features. Zootaxa, 4407 (4), 451-482. https://doi.org/10.11646/zootaxa.4407.4.1

Montaño-Moreno, H., Lucio-Palacio, C. R. (2019) Arácnidos. Pp. 173-180. In: La biodiversidad en San Luis Potosí: Estudio de estado, Vol. II. Comisión Nacional para el Conocimiento y Uso de la Biodiversidad (CONABIO), Universidad Autónoma de San Luis Potosí, Instituto Potosino de Investigación Científica y Tecnológica. Gobierno del estado de San Luis Potosí: Secretaria de Ecología y Gestión Ambiental, México.

Morrone, J. J. (2005) Hacia una síntesis biogeográfica de México. Revista mexicana de biodiversidad, 76 (2), 207-252.

Navarro-Rodríguez, C. I., Valdez-Mondragón, A. (2020) Description of a new species of Loxosceles Heineken \& Lowe (Araneae, Sicariidae) recluse spiders from Hidalgo, Mexico, under integrative taxonomy: morphological and DNA barcoding data (CO1 + ITS2). European Journal of Taxonomy, 704, 1-30. https://doi.org/10.5852/ejt.2020.704

Pickard-Cambridge, F. O. (1901) Arachnida - Araneida and Opiliones. Pp. 193-312. In: Biologia Centrali-Americana, Zoology, 2. London.

Pickard-Cambridge, F. O. (1902) Arachnida - Araneida. Pp. 305-316. In: Biologia CentraliAmericana, Zoology, 1. London.

Platnick, N. I. (2020) Spiders of the World. A natural history. Princeton University Press, USA, 256 pp. 
Platnick, N. I., Lau, A. (1975) A revision of the celer group of the spider genus Anyphaena (Araneae, Anyphaenidae) in Mexico and Central America. American Museum Novitates, 2575, 1-36.

Polis, G. A., Hurd, S. D. (1995) Extraordinarily high spider densities on islands: flow of energy from de marine to terrestrial food webs and the absence of predation. Proceedings of the National Academy of Sciences of USA, 92, 4382-4386.

Ponce, J. (2005) Insectos y arácnidos. Pp. 90-94. In: G. L. E. Villaseñor (Ed.). La biodiversidad en Michoacán: Estudio de Estado. Comisión Nacional para el Conocimiento y Uso de la Biodiversidad, Secretaría de Urbanismo y Medio Ambiente, Universidad Michoacana de San Nicolás de Hidalgo, México.

Rivera-Quiroz, F. A., Álvarez-Padilla, F. (2019) Description of five new Wulfila (Araneae, Anyphaenidae) species from Mexico with comments on the taxonomy of the genus. Zootaxa, 4712 (2), 269-289. https://doi.org/10.11646/zootaxa.4712.2.6

Roth, V. D. (1968) The spider genus Tegenaria in the Western Hemisphere (Agelenidae). American Museum Novitates, 2323, 1-33.

Salgueiro-Sepúlveda, F. J., Álvarez-Padilla, F. (2018) New species of the orb-weaving spider genus Chrysometa (Araneae, Tetragnathidae) from oak forests near of the Pico de Orizaba National Park (Veracruz, Mexico). Zootaxa, 4450 (3), 301-330. https://doi.org/10.11646/zootaxa.4450.3.1

Salas Suárez, N., Beutelspacher, C. R. (2011) Alacranes y Arañas. Pp. 168-173. In: Riqueza biológica de Quintana Roo: análisis para su conservación. Comisión Nacional para el Conocimiento y Uso de la Biodiversidad (CONABIO), Colegio de la Frontera Sur (ECOSUR), Gobierno del Estado de Quintana Roo y Programa de Pequeñas Donaciones-México, México.

Ubick, D., Paquin, P., Cushing, P. E., Roth, V. (Eds) (2017) Spiders of North America: an identification manual, 2nd Edition American Arachnological Society, Keene, New Hampshire, USA.

Valdez-Mondragón, A. (2013) Taxonomic revision of the spider genus Ixchela Huber, 2000 (Araneae: Pholcidae), with description of ten new species from Mexico and Central America. Zootaxa, 3608, 285-327. https://doi.org/10.11646/zootaxa.3608.5.1

Valdez-Mondragón, A. (2017) On the poorly known haplogynae spiders of the genus Ochyrocera Simon (Araneae, Ochyroceratidae) from Mexico: description of two new species with an updated identification key for Mexican species. Zootaxa, 4226 (2), 194-204. https://doi.org/10.11646/zootaxa.4226.2.2

Valdez-Mondragón, A. (2020) COI mtDNA barcoding and morphology for species delimitation in the spider genus Ixchela Huber (Araneae: Pholcidae), with the description of two new species from Mexico. Zootaxa, 4747 (1), 54-76.

https://doi.org/10.11646/zootaxa.4747.1.2

Valdez-Mondragón, A., Cortez-Roldán, M. R., Juárez-Sánchez, A. R., Solís-Catalán, K. P. (2018) A new species of Loxosceles Heineken \& Lowe (Araneae, Sicariidae), with updated distribution records and biogeographical comments for the species from Mexico, including a new record of Loxosceles rufescens (Dufour). ZooKeys, 802, 39-66. 
https://doi.org/10.3897/zookeys.802.28445

Valdez-Mondragón, A., Navarro-Rodríguez, C. I., Solís-Catalán, K. P., Cortez-Roldán, M. R., Juárez-Sánchez, A. R. (2019) Under an integrative taxonomic approach: the description of a new species of the genus Loxosceles (Araneae, Sicariidae) from Mexico City. ZooKeys, 892, 93-133.

https://doi.org/10.3897/zookeys.892.39558

World Spider Catalog (2021) World Spider Catalog. Version 21.5 Natural History Museum Bern. Disponible en: http://wsc.nmbe.ch (consultado 13 enero 2021). 